\title{
Tracking mesenchymal stem cell contributions to regeneration in an immunocompetent cartilage regeneration model
}

\author{
Daniela Zwolanek, ${ }^{1}$ María Satué, ${ }^{1}$ Verena Proell, ${ }^{1}$ José R. Godoy, ${ }^{1}$ Kathrin I. Odörfer, ${ }^{1}$ \\ Magdalena Flicker, ${ }^{1}$ Sigrid C. Hoffmann, ${ }^{2}$ Thomas Rülicke, ${ }^{1}$ and Reinhold C. Erben ${ }^{1}$ \\ 'Department of Biomedical Sciences, University of Veterinary Medicine, Vienna, Austria. ${ }^{2}$ Medical Research Center, Medical \\ Faculty Mannheim, University of Heidelberg, Mannheim, Germany.
}

It is currently controversially discussed whether mesenchymal stem cells (MSC) facilitate cartilage regeneration in vivo by a progenitor- or a nonprogenitor-mediated mechanism. Here, we describe a potentially novel unbiased in vivo cell tracking system based on transgenic donor and corresponding immunocompetent marker-tolerant recipient mouse and rat lines in inbred genetic backgrounds. Tolerance of recipients was achieved by transgenic expression of an immunologically neutral but physicochemically distinguishable variant of the marker human placental alkaline phosphatase (ALPP). In this dual transgenic system, donor lines ubiquitously express WT, heat-resistant ALPP protein, whereas recipient lines express a heat-labile ALPP mutant (ALPP ${ }^{E 451}$ ) resulting from a single amino acid substitution. Tolerance of recipient lines to ALPP-expressing cells and tissues was verified by skin transplantation. Using this model, we show that intraarticularly injected MSC contribute to regeneration of articular cartilage in full-thickness cartilage defects mainly via a nonprogenitor-mediated mechanism.

License: This work is licensed under the Creative Commons Attribution 4.0 International License. To view a copy of this license, visit http:// creativecommons.org/licenses/ by/4.0\%.

Authorship note: DZ, MS, and VP contributed equally to this work.

Conflict of interest: The authors have declared that no conflict of interest exists.

Submitted: February 26, 2016 Accepted: September 20, 2017 Published: October १९, 2017

\section{Reference information:} JCI Insight. 2017;2(20): e87322. https://doi.org/10.1172/jici. insight.87322.

\section{Introduction}

Mesenchymal stem cells (MSC) are multipotent cells isolated from adult BM or other tissues. MSC can be easily isolated, expanded, and differentiated into multiple cell lineages such as bone, cartilage, muscle, or fat tissue under defined culture conditions. Adult MSC are currently being evaluated for a wide number of cell-based therapeutic approaches, such as skeletal tissue regeneration, myocardial regeneration, and treatment of graft-versus-host disease (1).

Although several studies investigating cartilage regeneration using intraarticularly injected MSC reported promising results in different model systems (2-6), it is currently controversially discussed whether the positive therapeutic effects of MSC are due to a progenitor or a nonprogenitor function of injected cells (7). It is unclear whether MSC injected into a joint attach to cartilage lesions, divide, differentiate, and give rise to new MSC-derived chondrocytes, or whether they just orchestrate regeneration by a nonprogenitor function, e.g., by secreting factors stimulating host cells to promote regeneration. This controversy has been fueled by the lack of appropriate translational model systems that are able to unequivocally answer this key question. We have recently shown that genetically labeled MSC are able to attach to cartilage lesions via a $\beta_{1}$ integrin-mediated mechanism in vitro (8), but it is generally believed that, in vivo, only few of the intraarticularly injected cells adhere to damaged cartilage and may subsequently participate in tissue regeneration.

In order to gain more mechanistic insight into the fate and function of therapeutically administered MSC, it is necessary to reliably track the injected cells. The most widely used method to label cells is the introduction of a stable genetic marker that is expressed in the whole progeny of a specific cell. Therefore, use of cells from transgenic donor animals is probably the most robust way of exploring the long-term fate of transplanted cells. However, a major problem in this context is immune-mediated rejection of cells labeled with immunogenic marker proteins in immunocompetent hosts. Most of the current markers for cell tracking — such as fluorescent proteins, the bacterial enzyme LacZ, firefly 
luciferase, Herpes simplex thymidine kinase, or human placental alkaline phosphatase (ALPP) - are foreign, immunogenic proteins. In recent years, it has become increasingly clear that membrane or even intracellular expression of any foreign protein, and thus of any marker protein, elicits immune-mediated rejection of transplanted nonmalignant cells expressing the marker in an immunocompetent recipient (9-13). This problem has hampered the usefulness of cell tracking models in regenerative medicine, especially in long-term studies. To date, it has been circumvented by the use of immunodeficient animals such as $P r k d c^{\text {scid }}$ (severe combined immunodeficiency) mice or by drug- and irradiation-based immunosuppression (14). These strategies obviously create artificial systems that may not be predictive of the situation in a human patient because an intact host immune system is a very important component in the course of many, if not all, diseases.

The animal model described in the current report aims to fill this translational gap. Here, we describe a transgenic marker-tolerant animal model in mice and rats that allows tracing the fate of genetically labeled cells in the complete absence of immune-mediated rejection in immunocompetent hosts. We have previously introduced the concept of marker tolerance using neonatal tolerization to a specific marker protein (15). The current model is based on a dual transgenic system, consisting of a donor line and a corresponding marker-tolerant recipient line characterized by innate tolerance to the donor marker. As proof of concept, we used the marker ALPP for its convenience of detection in hard tissues. Using this novel in vivo cell tracking model, we show that intraarticularly injected MSC contribute to healing of a focal full-thickness cartilage defect mainly by a nonprogenitor effect.

\section{Results}

Generation of transgenic mouse lines. For our proof-of-principle experiments, we employed ALPP as marker due to its ease of detection in soft (16-18) and hard tissues (19) and because ALPP is known to be developmentally neutral in mice and rats (16) and does not influence the behavior of ALPP-expressing MSC (20). In this dual transgenic system, the donor line $\operatorname{Tg}(\mathrm{ALPP})$ expresses WT ALPP in a ubiquitous fashion and serves as an unlimited source of genetically labeled cells and tissues. To generate ALPP-tolerant recipients, we made use of ALPP's unique heat stability in comparison with other human alkaline phosphatases (APs). Human APs are highly homologous; for example, the heat-labile germ cell AP (GCAP) differs only in 7 amino acid residues from heat-stable ALPP (21). It has previously been shown that amino acid position 451 is crucial for conveying heat stability in human APs (22). Based on these experiments, it seemed possible that changing glutamic acid 451 to glycine might be sufficient to render the protein heat labile. The heat-labile form of ALPP could easily be distinguished from WT ALPP by heat inactivation, similar to endogenous APs. We hypothesized further that the immune system of transgenic mice and rats expressing $\mathrm{ALPP}^{\mathrm{E} 451 \mathrm{G}}\left(\mathrm{Tg}\left[\mathrm{ALPP}^{\mathrm{E} 451 \mathrm{G}}\right]\right)$ might fail to recognize WT ALPP as a foreign protein because the heat-sensitive derivative $\mathrm{ALPP}^{\mathrm{E} 451 \mathrm{G}}\left(\mathrm{ALPP}^{\mathrm{m}}\right)$ is virtually identical to native ALPP.

To test this idea, we generated donor and recipient mouse and rat lines in a C57BL/6N and Fischer 344 inbred background, respectively, by pronuclear injection of mouse and rat zygotes and random integration of the transgene. Driven by a truncated Rosa26 promoter (23), donor lines express WT ALPP, whereas recipient lines express ALPP ${ }^{\mathrm{E} 51 \mathrm{G}}$ (Figure 1, A and B). Transgenic founders were identified by PCR and Southern blotting on genomic tail DNA (data not shown). Transcription and translation of the transgenes were verified by reverse transcription PCR (RT-PCR) on cDNA (Figure 1C) and by the immunoblotting of protein lysates prepared from lungs of donor and recipient mice (Figure 1D) or lysates prepared from kidneys of donor and recipient rats (Figure 1E). Donor lines were selected for high ALPP expression, whereas $\operatorname{Tg}\left(\mathrm{ALPP}^{\mathrm{E} 451 \mathrm{G}}\right)\left(\mathrm{Tg}\left[\mathrm{ALPP}^{\mathrm{m}}\right]\right)$ recipients were selected for low expression to facilitate heat inactivation and to reduce any potential background. It is important to note that the anti-ALPP antibody (SP15) used for immunoblotting does not distinguish between ALPP and ALPP ${ }^{m}$ because the proteins differ only in one amino acid. Two donor and 3 recipient mouse lines, as well as 1 donor and 1 recipient rat line, were chosen for further analysis. The data in this report focus on the donor line C57BL/6N-Tg(R26-ALPP)181Biat (Tg[ALPP]) and the recipient lines C57BL/6N-Tg(R26-ALPP $\left.{ }^{\mathrm{E} 451 \mathrm{G}}\right)$ 192Biat (Tg[ALPP $\left.\left.{ }^{\mathrm{m}}\right]\right)$ and F344-Tg(R26-ALPP $\left.{ }^{\mathrm{E} 451 \mathrm{G}}\right)$ 545Mhm (Tg[ALP$\left.\left.\mathrm{P}^{\mathrm{m}}\right]\right)$. All results reported for the C57BL/6N-Tg(ALPP $\left.{ }^{\mathrm{m}}\right) 192 \mathrm{Biat}$ recipient line were confirmed in two other selected recipient lines (data not shown). Because our self-produced F344-Tg(ALPP) donor line expressed the ALPP transgene at lower levels compared with the F344-Tg(ALPP) rat line generated by Kisseberth and coworkers (16), we used the latter rat line as a donor line for all subsequent experiments. 
A

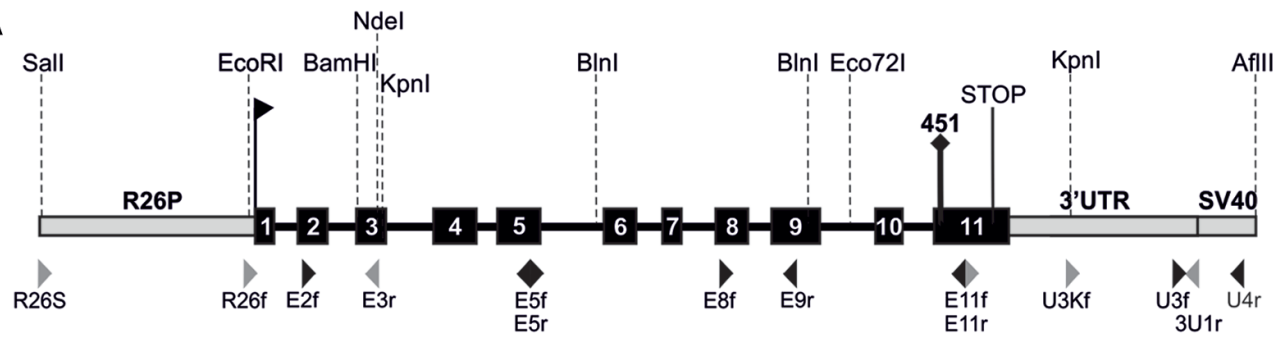

B

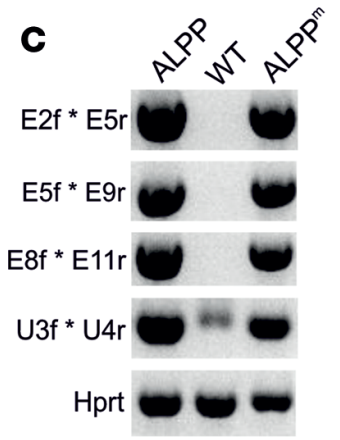

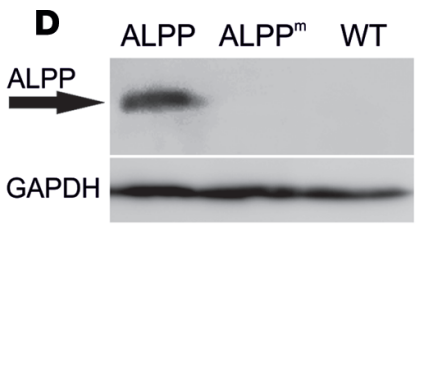

Figure 1. Structure of human placental alkaline phosphatase (ALPP) transgenic constructs and transgene expression in transgenic mouse and rat lines. (A) Structure of transgenic constructs for pronuclear injection, including relevant endonuclease restriction sites and primer positions. A point mutation in codon 451 of the ALPP gene introduced by site-directed mutagenesis results in an $\mathrm{E} 451 \mathrm{C}$ substitution, giving rise to the heat-labile ALPPE451C (ALPPm) derivative. (B) Variation in amino acid sequence of heat-stable WT ALPP and heat-labile ALPPm. (C) Transcription of full-length WT and mutated ALPP was verified by RT-PCR on lung cDNA with amplicons spanning the whole transcript, exemplarily shown for the mouse model. $n \geq 7$ per genotype. (D and $\mathbf{E}$ ) Protein expression was examined by immunoblotting of mouse lung (D, $n=4$ per genotype) and rat kidney tissue ( $E, n=4$ per genotype), showing high ALPP expression in ALPP-transgenic donor lines but no ALPP/ALPPm protein expression in ALPP ${ }^{m}$-transgenic and WT mice. GAPDH was used as loading control.

Heat sensitivity of mutant $A L P P$ and tolerance of $T g\left(A L P P^{n}\right)$ recipients to WT ALPP. We initially tested the heat stability of $\mathrm{ALPP}^{\mathrm{m}}$. Figure $2 \mathrm{~A}$ and Supplemental Figure 1, A and B (supplemental material available online with this article; https://doi.org/10.1172/jci.insight.87322DS1) show that, similar to endogenous APs, heat pretreatment of paraffin or plastic sections at $72^{\circ} \mathrm{C}$ for 35 minutes completely inactivates ALPP ${ }^{\mathrm{m}}$ but not WT ALPP. Thus, tissues from $\operatorname{Tg}\left(\mathrm{ALPP}^{\mathrm{m}}\right)$ recipients do not show histochemical background staining after heat inactivation.

We next examined the tolerance of $\operatorname{Tg}\left(\mathrm{ALPP}^{\mathrm{m}}\right)$ recipient mice and rats to tissues from $\mathrm{Tg}(\mathrm{ALPP})$ donors by monitoring skin graft survival over 24 weeks in mice and over 4 weeks in rats. Autotransplantations and WT-to-WT transplantations served as controls. All transplantations were performed in a sex-matched manner. Macroscopic appearance and graft scoring of skin transplants from $\operatorname{Tg}(\mathrm{ALPP})$ mice into $\operatorname{Tg}(\mathrm{ALPP})$ recipients was indistinguishable from autotransplants and WT-to-WT transplants (Supplemental Figure 2, A and $\mathrm{B}$ ). In accordance with the finding that chronic rejection may not be clearly evident at the macroscopic level in inbred mice (24), skin grafts from $\operatorname{Tg}(\mathrm{ALPP})$ mice transplanted into WT mice of the same background were not visibly rejected over the time course of the experiment (Supplemental Figure 2, A and B). Similarly, macroscopic appearance and scoring of skin transplants from $\mathrm{Tg}(\mathrm{ALPP})$ rats into $\mathrm{Tg}\left(\mathrm{ALPP} \mathrm{P}^{\mathrm{m}}\right)$ rats were equivalent to autotransplantation and WT-to-WT transplants (Supplemental Figure 2, C and D). In contrast, skin grafts from $\mathrm{Tg}(\mathrm{ALPP})$ rats were clearly rejected in otherwise isogenic WT recipients within 4 weeks after transplantation, resulting in a significantly higher score (Supplemental Figure 2D).

Histochemical staining for ALPP activity showed strong staining of grafts from Tg(ALPP) donors in $\operatorname{Tg}\left(\mathrm{ALPP}^{\mathrm{m}}\right)$ recipient mice at 3 and 24 weeks after transplantation (Figure $2 \mathrm{~B}$ and Supplemental Figure $\left.1 \mathrm{C}\right)$. It is noteworthy that ALPP staining was distinctly reduced as early as 3 weeks after transplantation in WT compared with $\operatorname{Tg}\left(\mathrm{ALPP}^{\mathrm{m}}\right)$ recipient mice. In agreement with the results obtained in mice, skin grafts from $\operatorname{Tg}(\mathrm{ALPP})$ donor rats showed strong histochemical ALPP staining in $\operatorname{Tg}\left(\mathrm{ALPP}^{\mathrm{m}}\right)$ recipient rats, but ALPP staining was almost absent in WT recipients 4 weeks after transplantation (Figure 2D and Supplemental Figure 1E).

To examine graft rejection in more detail, we quantified leukocyte infiltration in tissue sections of allo- and autotransplants at 3 and 24 weeks after surgery by anti-CD45R and anti-CD45 IHC in mice and rats, respectively. In accordance with the data obtained from ALPP histochemistry, skin grafts from $\operatorname{Tg}$ (ALPP) donors showed considerable influx of immune cells at 3 and 24 weeks after transplantation in WT recipient mice (Figure 2C and Supplemental Figure 1D) and at 4 weeks after transplantation in WT 


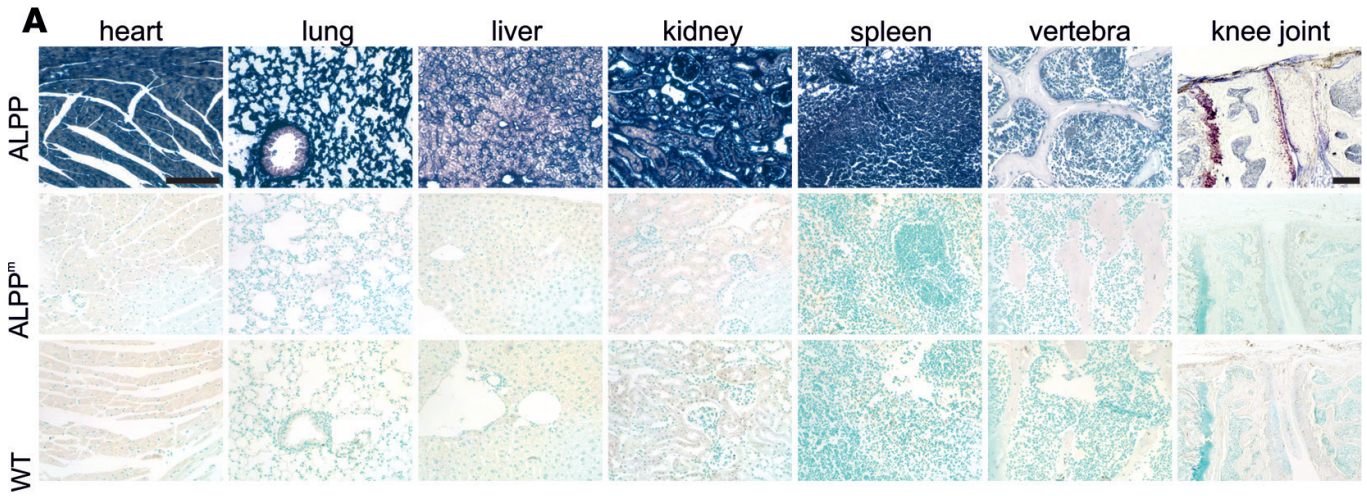

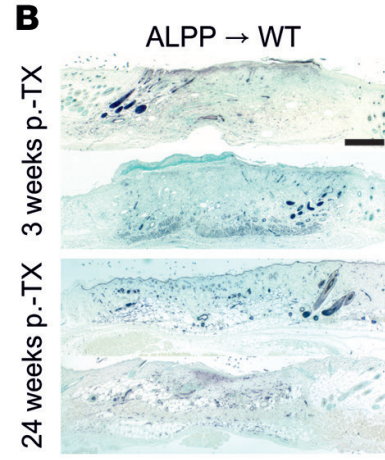

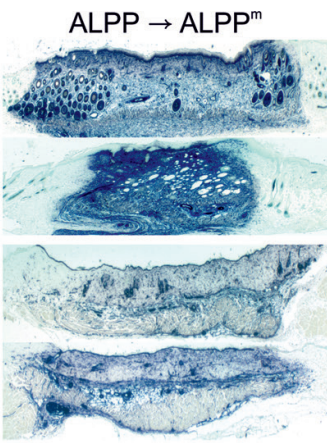

D

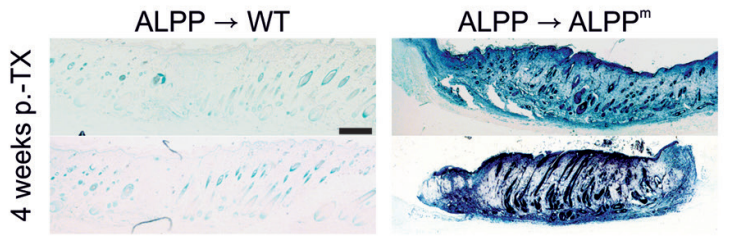

C
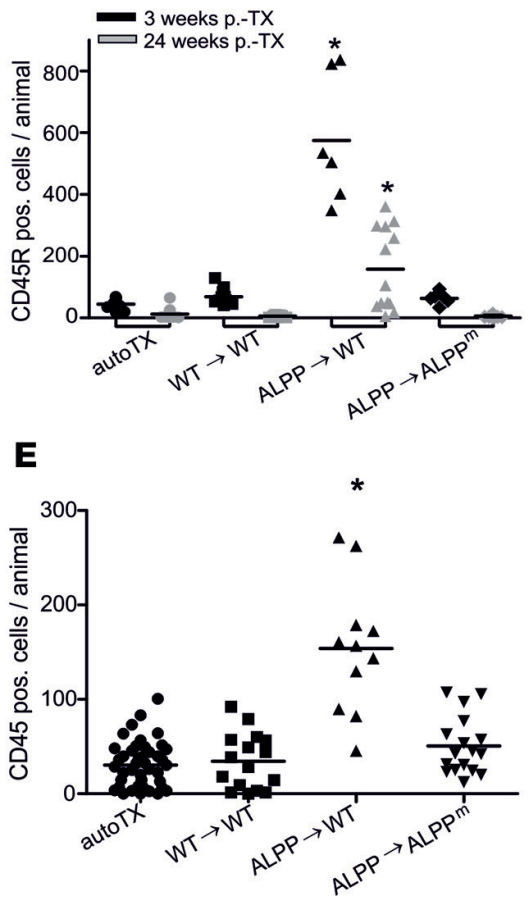

Figure 2. Histochemical ALPP staining and long-term tolerance of $\operatorname{Tg}\left(\mathbf{A L P P}{ }^{\mathrm{m}}\right)$ recipients to skin grafts from $\operatorname{Tg}(\mathbf{A L P P})$ donors. (A) ALPP histochemistry on paraffin or plastic (bone and knee joint) sections from various organs of $\mathrm{Tg}(\mathrm{ALPP}), \mathrm{Tg}(\mathrm{ALPPm})$, and WT mice after a 35 -minute heat inactivation at $72^{\circ} \mathrm{C}$. $\mathrm{Tg}$ (ALPP) donors show strong staining in all tissues, whereas no enzyme activity could be detected in WT and Tg(ALPPm) mice. Scale bar: $50 \mu \mathrm{m}$. $n=10$ per group. (B) Histochemistry of skin grafts from Tg(ALPP) at 3 and 24 weeks after transplantation. Strong ALPP staining was present in Tg(ALPP) grafts transplanted into $\mathrm{Tg}\left(\mathrm{ALPP} \mathrm{m}^{\mathrm{m}}\right.$ ) recipients. Grafts from $\mathrm{Tg}(\mathrm{ALPP})$ mice were rejected by WT recipients, shown by the pronounced decline in ALPP staining within the grafts over time. Scale bar: $500 \mu \mathrm{m} . n=10$ per group. (C) Leukocyte infiltration of skin grafts from $\mathrm{Tg}(\mathrm{ALPP})$ donor mice was quantified by CD45R immunostaining at 3 and 24 weeks after surgery and expressed as number of positive cells per tissue section. $n \geq 5$ per group. (D) Histochemistry of skin grafts from $\mathrm{Tg}(\mathrm{ALPP})$ rats at 4 weeks after transplantation. Strong ALPP staining was present in $\mathrm{Tg}(\mathrm{ALPP})$ grafts transplanted into $\mathrm{Tg}\left(\mathrm{ALPP} \mathrm{m}^{\mathrm{m}}\right)$ recipients. Grafts from $\mathrm{Tg}(\mathrm{ALPP})$ rats were rejected by WT recipients, as shown by the almost absent ALPP staining within the grafts. Scale bar: $500 \mu \mathrm{m}$. $n=10$ per group. (E) Leukocyte infiltration of skin grafts from $\mathrm{Tg}(\mathrm{ALPP})$ donor rats was quantified by CD45 immunostaining at 4 weeks after transplantation and expressed as number of positive cells per tissue section. $n \geq 10$ per group. ${ }^{*} P<0.05$ by one-way ANOVA followed by Student-Newman-Keuls multiple comparison test. ALPP, human

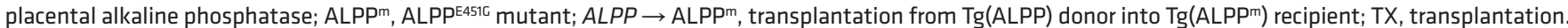

rats (Figure 2E and Supplemental Figure 1F). In contrast, leukocyte infiltration of the ALPP transgenic grafts did not occur in $\mathrm{Tg}\left(\mathrm{ALPP}^{\mathrm{m}}\right)$ recipients at any time point after transplantation, demonstrating longterm tolerance of $\operatorname{Tg}\left(\mathrm{ALPP}^{\mathrm{m}}\right)$ recipient mice and rats to tissues from ALPP transgenic donors (Figure 2, $\mathrm{C}$ and $\mathrm{E}$ and Supplemental Figure 1, D and F).

MSC attach to full-thickness cartilage defects but do not differentiate into mature chondrocytes. We next examined the ability of this cell tracking model to answer an unresolved question in regenerative medicine, namely whether intraarticularly injected MSC augment cartilage regeneration via a progenitor- or 

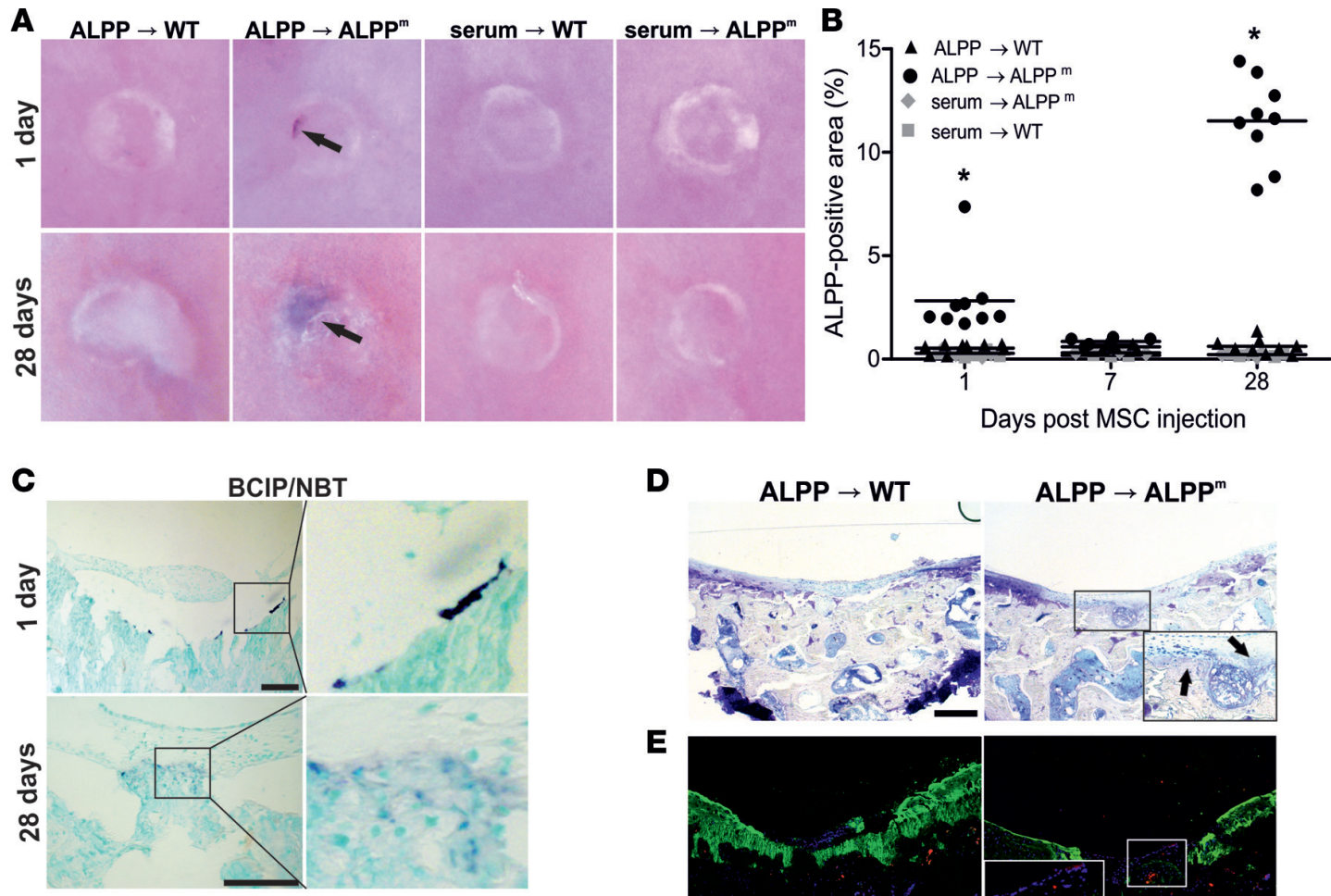

E
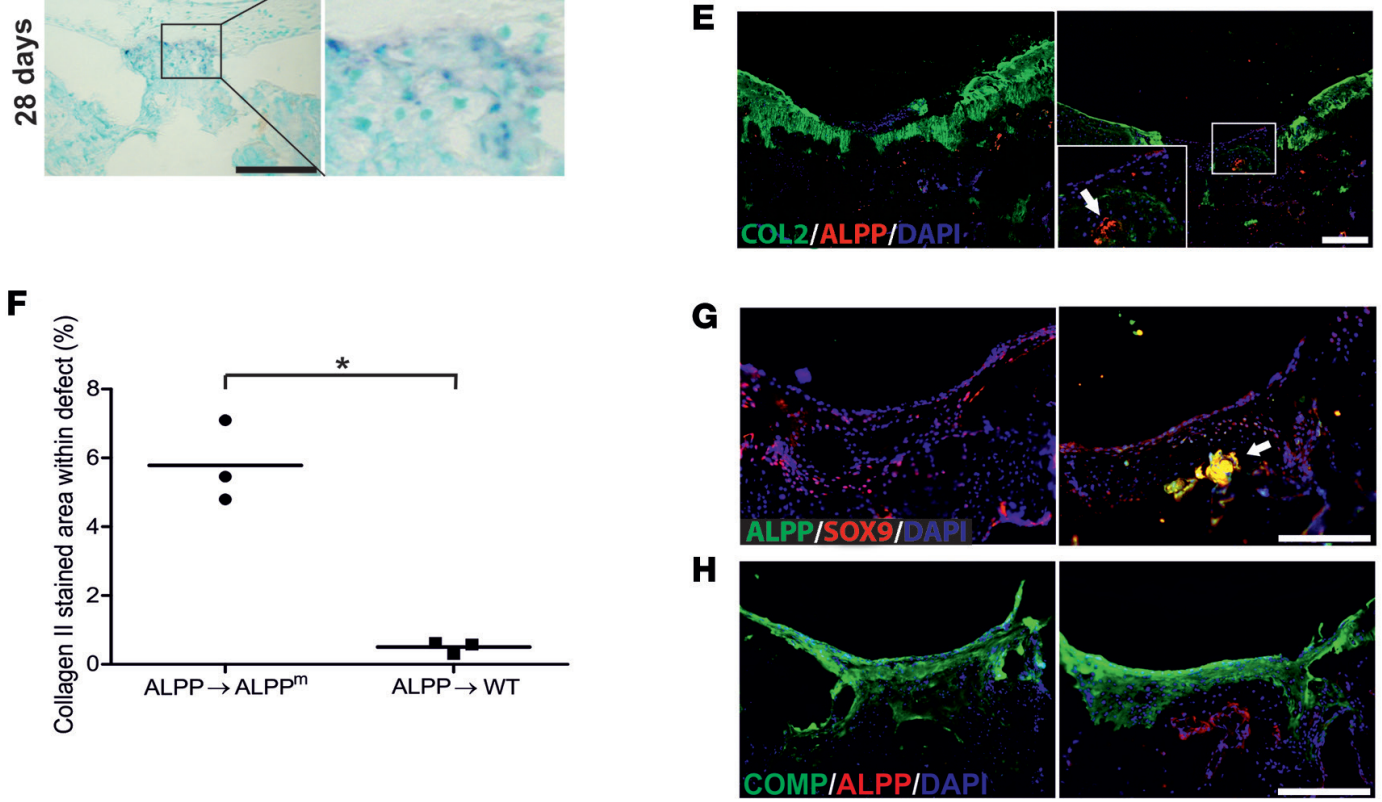

Figure 3. Intraarticularly injected MSC contribute to cartilage regeneration in a rat model of full-thickness cartilage defects. (A) Histochemical ALPP staining of the distal femoral joint surface with a full-thickness cartilage defect in the patellar groove 1 and 28 days after intraarticular injection of serum or $1 \times 10^{7} \mathrm{MSC}$ from Tg(ALPP) donor rats. Arrows show ALPP-positive staining in the defects. ALPP staining was absent in WT or $\operatorname{Tg}(\mathrm{ALPP})$ recipients injected with MSC from $\operatorname{Tg}(\mathrm{ALPP})$ donors or serum, respectively. Scale bar: $250 \mu \mathrm{m}$. (B) Quantification of the ALPP-positive area within the full-thickness defects. Dots represent ALPP-positive area per animal. $n \geq 5$ per group. ${ }^{*} P<0.05$, ALPP $\rightarrow$ ALPPm recipients vs. all other groups by Kruskal-Wallis test followed by Mann-Whitney $U$ test. (C) Histochemical ALPP staining of cryosections from femoral full-thickness cartilage defects 1 and 28 days after injection of $1 \times 10^{7}$ MSC from Tg(ALPP) donor rats into the knee of Tg(ALPPm) recipients. Insets show ALPP-positive cells at the bottom of the defects. Scale bar: $50 \mu \mathrm{m} . n=3$ animals per group. (D) Toluidine blue staining of cryosections from full-thickness cartilage defects, 28 days after injection of $1 \times 10^{7}$ MSC from Tg(ALPP) donor rats into WT or Tg(ALPPm) recipients. Black arrows indicate induction of neocartilage formation within the defects of $\mathrm{Tg}(\mathrm{ALPP})$ ) animals. Scale bar: $50 \mu \mathrm{m} . n=3$ per group. (E, G, and $\mathbf{H})$ Immunofluorescence staining of cryosections from full-thickness cartilage defects using anti-collagen II (anti-COL2, green) and anti-ALPP antibodies (red) (E), anti-ALPP (green) and anti-Sox9 antibodies (red) (G), or anti-COMP (green) and anti-ALPP antibodies (red) (H) 1 month after injection of $1 \times 10^{7}$ MSC from $\mathrm{Tg}(\mathrm{ALPP})$ donor rats into WT or Tg(ALPPm) recipients. White arrows in insets show ALPP-expressing cells at the bottom of the defect surrounded by COL2-containing matrix (E) and ALPP- and SOX9-coexpressing cells within the defect (G) in Tg(ALPPm) recipients. Neither ALPP-expressing cells, new COL2 matrix, nor ALPP- SOX9-coexpressing cells were found in WT recipients. Scale bar $=50 \mu \mathrm{m}$. $n=3$ per group. (F) Quantification of the COL2-stained area within the defects in cryosections 28 days after injection of MSC from $\operatorname{Tg}(A L P P)$ donors in WT or Tg(ALPPm) recipients. Dots represent means of COL2-stained area per animal. $n=3$ per group. ${ }^{*} P<0.05$ by Student's $t$ test. ALPP, human placental alkaline phosphatase; ALPPm, $A L P P^{E 451 C}$ mutant; $A L P P \rightarrow A L P P m$, transplantation from $\operatorname{Tg}(A L P P)$ donor into $\mathrm{Tg}\left(\mathrm{ALPP}{ }^{\mathrm{m}}\right)$ recipient; $\mathrm{TX}$, transplantation. 
nonprogenitor-mediated mechanism. To this end, we established a focal cartilage defect model in rats. Full-thickness cartilage defects with a diameter of $0.7 \mathrm{~mm}$ were generated in the patellofemoral groove of the right knee in female $\mathrm{Tg}\left(\mathrm{ALPP}^{\mathrm{m}}\right)$ and WT rats. The left intact knee served as internal control. Two weeks after surgery, we injected $1 \times 10^{7} \mathrm{MSC}$ isolated from sex-matched $\mathrm{Tg}(\mathrm{ALPP})$ donor rats or vehicle (serum) into the left and right knees. We used native rat serum for suspension of MSC to facilitate attachment to the defects (8). A detailed characterization of the MSC population has been reported earlier (20).

Histochemical analysis of ALPP activity directly after femur isolation demonstrated that ALPPexpressing MSC were able to successfully attach to the full-thickness defects in $\operatorname{Tg}(\mathrm{ALPP})$ recipients, whereas no attachment was seen in WT recipients or in vehicle controls 1 day after injection (Figure 3, A-C). Seven days after injection, only few ALPP-positive cells were found in the defects of $\operatorname{Tg}(\operatorname{ALPP})^{\mathrm{m}}$. Interestingly, however, 28 days after injection, a significant area of the defects was covered by ALPPpositive cells in $\operatorname{Tg}\left(\mathrm{ALPP}^{\mathrm{m}}\right)$ recipients, whereas no ALPP staining was found in transplanted WT animals or serum-injected controls (Figure 3, A and B).

In order to follow up the intriguing finding that ALPP-expressing MSC were absent in cartilage defects of WT recipients as early as one day after injection, we analyzed synovial ALPP staining and the amount of ALPP present in the joint capsule tissue by histochemical staining and immunoblotting, respectively, 1 day after injection of MSC from $\mathrm{Tg}$ (ALPP) donors. As expected, ALPP staining and ALPP protein expression was only found in the joint capsule of knees injected with ALPP-expressing MSC but not in serum-injected WT and $\operatorname{Tg}\left(\mathrm{ALPP}^{\mathrm{m}}\right)$ controls (Supplemental Figure 3, A and B). Surprisingly, however, the synovial ALPP-stained area, as well as the abundance of ALPP protein, was higher in the joint capsule of $\operatorname{Tg}\left(\mathrm{ALPP}^{\mathrm{m}}\right)$ compared with WT recipients 1 day after injection of MSC from $\operatorname{Tg}(\mathrm{ALPP})$ donors (Supplemental Figure 3, A and C). It may be argued that the higher ALPP protein expression in $\operatorname{Tg}\left(\mathrm{ALPP}^{\mathrm{m}}\right)$ compared with WT recipient mice could be due to background expression of ALPP ${ }^{\mathrm{m}}$ because the anti-ALPP antibody (SP15) used for immunoblotting does not distinguish between ALPP and ALPPm. However, as shown in Supplemental Figure 3B, the low background expression of ALPPm in $\operatorname{Tg}\left(\operatorname{ALPP}{ }^{\mathrm{m}}\right)$ rats is detected by immunoblotting only when very different amounts of protein are loaded (right lane in Supplemental Figure 3B). To find a possible explanation for the rapid reduction of ALPP abundance in WT hosts, we analyzed neutrophil activation by examining myeloperoxidase (MPO) expression as an indicator for activation of the innate immune system (25). Although the changes were not statistically significant, WT recipient joint-capsule homogenates showed a tendency for higher MPO expression compared with $\operatorname{Tg}\left(\mathrm{ALPP}^{\mathrm{m}}\right)$ recipients (Supplemental Figure 3D). These findings may suggest that injection of ALPP-expresssing MSC into the knee joint of WT recipients elicits a rapid activation of the innate immune system, resulting in rejection of the injected cells.

To examine the fate of injected MSC, we performed ALPP staining and immunofluorescence analysis of cryosections prepared from the undecalcified defects, using the newly developed tape method (26). ALPP-positive cells were found at the bottom of the defects at 1 and 28 days after injection in $\operatorname{Tg}(\mathrm{ALPP})$ recipients treated with MSC from $\mathrm{Tg}(\mathrm{ALPP})$ donors (Figure $3 \mathrm{C}$ ). In contrast, no ALPP-expressing cells were observed in WT recipients injected with ALPP-expressing MSC (Figure 3E) or in WT or Tg(ALPP $)$ rats injected with serum (Supplemental Figure 4, C, E, and G). Toluidine blue staining indicated beginning neocartilage formation only in $\operatorname{Tg}\left(\mathrm{ALPP}^{\mathrm{m}}\right)$ recipients treated with MSC from $\mathrm{Tg}(\mathrm{ALPP})$ donors but not in WT rats treated with ALPP-expressing MSC or in rats injected with serum (Figure 3D and Supplemental Figure 4A). In line with this finding, collagen II (COL2) expression within the defects was almost exclusively found in $\operatorname{Tg}\left(\mathrm{ALPP}^{\mathrm{m}}\right)$ recipients injected with ALPP-expressing MSC, 1 month after treatment (Figure $3, \mathrm{E}$ and $\mathrm{F}$ ). COL2 is a prominent cartilage extracellular matrix protein and indicates chondrogenic cell differentiation within the defects. ALPP-positive cells were found at the bottom of the defects surrounded by a matrix containing COL2 in $\mathrm{Tg}\left(\mathrm{ALPP}^{\mathrm{m}}\right)$ recipients (Figure $\left.3 \mathrm{E}\right)$. Interestingly, the early chondrogenic marker SRY-Box9, SOX9, was highly expressed by some ALPP-expressing cells at the bottom of the defects, 1 month after injection (Figure $3 \mathrm{G}$ ). Another prominent cartilage protein, the cartilage oligomeric matrix protein (COMP) was detectable in all defects (Figure 3H and Supplemental Figure 4G).

To analyze the long-term fate of injected MSC and their ability to support cartilage regeneration, we performed an additional 6-month experiment. Toluidine blue staining showed that the cartilage defect was almost fully regenerated in $\mathrm{ALPP}^{\mathrm{m}}$ recipients, 6 months after MSC injection (Figure 4A). In contrast, the defects were mostly filled with bone and covered only with a very thin layer of cartilage in WT animals injected with MSC (Figure 4, A-E) or in animals injected with serum (Supplemental Figure 4, B, D, F, and H). 
A

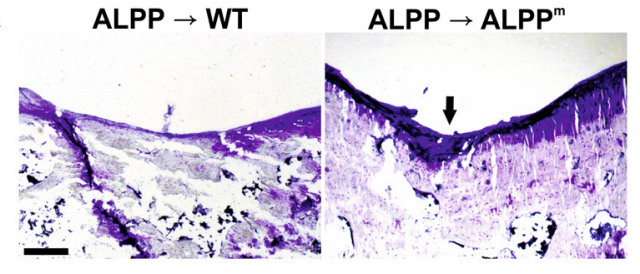

C

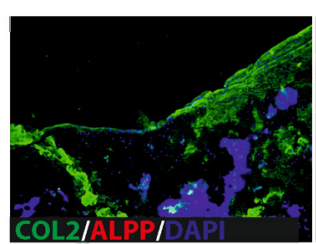

$\mathbf{E}$

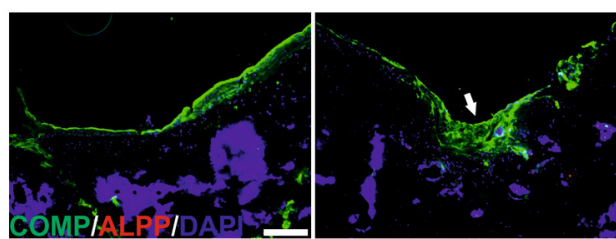

B

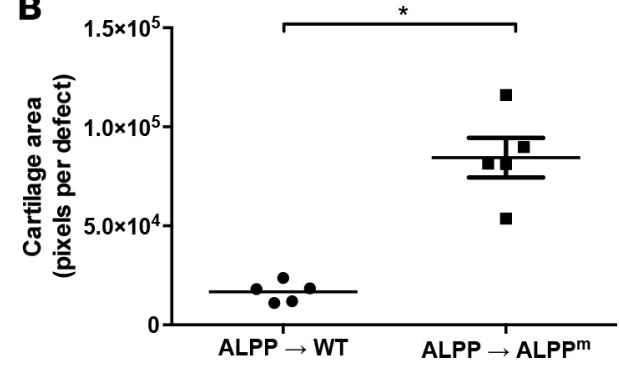

D

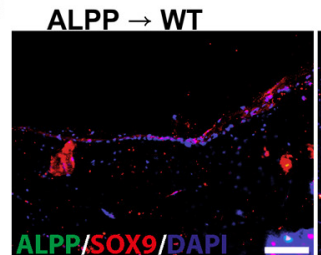

$A L P P \rightarrow$ ALPP $^{m}$

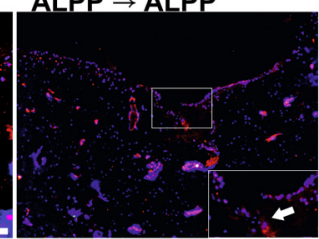

Figure 4. Intraarticularly injected MSC contribute to long-term cartilage regeneration mainly by a nonprogenitor-mediated mechanism. (A) Toluidine blue staining of cryosections from full-thickness cartilage defects 6 months after injection of $1 \times 10^{7} \mathrm{MSC}$ from Tg(ALPP) donor rats into WT or Tg(ALPPm) recipients. Black arrow shows neocartilage formation within the defect of $\mathrm{Tg}\left(\mathrm{ALPP} \mathrm{P}^{\mathrm{m}}\right.$ ) animals. Scale bar: $50 \mu \mathrm{m}$. $n=5$ per group. (B) Quantification of newly formed cartilage at the defect site, measured with Image). $n=5$ per group. ${ }^{*} P<0.05$ by Student's $t$ test. (C-E) Immunofluorescence staining of cryosections from full-thickness cartilage defects, using anti-collagen II (anti-COL2, green) and anti-ALPP antibodies (red) (C), anti-ALPP (green) and anti-SOX9 antibodies (red) (D), or anti-COMP (green) and anti-ALPP antibodies (red) (E) 6 months after injection of $1 \times 10^{7}$ MSC from Tg(ALPP) donor rats into WT or $\mathrm{Tg}\left(\mathrm{ALPP}{ }^{\mathrm{m}}\right)$ recipients. White arrows in inset show COL2-containing cartilaginous matrix (C), as well as SOX9 (D) and COMP (E) expression in the newly formed cartilage in $\mathrm{Tg}(\mathrm{ALPP})$ recipients. No ALPP-expressing cells were found in $\operatorname{Tg}(\mathrm{ALPP})$ ) and WT recipients. Scale bar: $50 \mu \mathrm{m}$. $n=5$ per group. ALPP, human placental alkaline phosphatase; ALPPm, ALPPE451 mutant; ALPP $\rightarrow$ ALPPm, transplantation from $\mathrm{Tg}(\mathrm{ALPP})$ donor into Tg(ALPPm) recipient.

The newly formed cartilage in $\operatorname{Tg}\left(\mathrm{ALPP}^{\mathrm{m}}\right)$ recipients showed COL2, SOX9, and COMP expression, yet COL2 expression was still lower compared with the surrounding articular cartilage outside the defect (Figure 4, C-E). However, ALPP-positive cells were completely absent in $\mathrm{Tg}\left(\mathrm{ALPP}{ }^{\mathrm{m}}\right)$ recipients, 6 months after MSC injection (Figure 4, C-E), demonstrating that the regenerated cartilage was exclusively host derived. Taken together, these findings indicate that MSC locally attached to the defect contribute to cartilage regeneration, possibly by secreting bioactive factors that suppress chondrocyte hypertrophy and subsequent bone formation but do not give rise to new mature chondrocytes for long-term cartilage repair.

\section{Discussion}

We present a potentially novel in vivo cell tracking system that allows the fate of ALPP-labeled cells and tissues in immunocompetent recipients to be followed over long periods of time in the complete absence of immune-mediated rejection. The model is almost fully equivalent to the situation in human patients treated with autologous cells and may set new standards in the preclinical testing of regenerative therapies. Using this model, we show in a focal cartilage defect model that intraarticularly injected MSC contribute to longterm cartilage regeneration mainly through a nonprogenitor-mediated mechanism.

Earlier studies have shown that immunocompetent C57BL/6 mice rejected skin grafts from eGFP transgenic C57BL/6 mice within 100 days in 14 of 17 animals (27). In another report, only 8 of 66 skin grafts from GFP transgenic C57BL/ 6 mice were successfully engrafted over 6 months after transplantation in WT C57BL/6 mice (28). In line with these results, leukocyte infiltration of ALPP-expressing grafts in WT recipient mice and rats clearly indicated the presence of chronic rejection in our study, although macroscopic rejection of skin grafts from $\mathrm{Tg}(\mathrm{ALPP})$ mice in WT C57BL/6 recipients was not uniformly evident. Collectively, our data and the work of others suggests that, despite an inbred genetic background, cells or tissues expressing immunogenic marker proteins are invariably rejected in nontolerant immunocompetent hosts, irrespective of whether intracellular marker proteins such as GFP or membrane proteins such as ALPP are used. Moreover, the strong infiltration of ALPP transgenic skin grafts with immune cells at 3 and 4 weeks after transplantation in WT recipient mice and rats, respectively, indicates that immune-mediated 
rejection of cells and tissues labeled with immunogenic markers in nontolerant immunocompetent hosts is not only a problem in long-term studies, but also a confounding factor in short-term experiments.

Leukocyte infiltration of ALPP-expressing skin grafts was completely absent in $\operatorname{Tg}(\mathrm{ALPP})$ recipient mice and rats. Thus, the strategy of inducing innate tolerance to a marker protein by expression of a mutant marker protein differing in a single amino acid position proved successful. Our results suggest that ALPP and $A L P P^{\mathrm{E} 451 \mathrm{G}}$ are indeed immunologically neutral (i.e., the murine and rat immune systems do not distinguish between the 2 variants of the protein). For our proof-of-concept studies we used ALPP as a marker protein because of its ease of detection in histological sections $(15,19,29)$ and its suitability for electron microscopic analysis (30). It is likely that a similar approach (i.e., site-directed mutagenesis of single or few amino acids in key positions of the molecule) will also be suitable for other marker proteins such as fluorescent proteins, luciferase, thymidine kinase, or LacZ.

The strategy of inducing tolerance to a marker protein by transgenic overexpression of an immunologically neutral but physicochemically distinguishable variant of the marker has two other important advantages. Firstly, because transgenes can also efficiently be introduced into the genome of rabbits (31), pigs (32), or monkeys (33), it is very likely that the same system can be employed to generate marker-tolerant tracking models in other species used as animal models. Even in species where isogeneic strains are not available, such as in pigs or monkeys, autologous cells harvested from transgenic marker-tolerant individuals and transfected with the WT marker could be tracked in the absence of immune-mediated rejection. Secondly, because both donor and recipient animals are active as hemizygous genotypes, our system can rapidly be employed to track cells from $\mathrm{KO}$ or knockin mouse models in the same genetic background.

An intriguing observation in our study was that injection of ALPP-expressing MSC into the knee joint of WT recipients elicits a rapid activation of the innate immune system, resulting in rejection of the injected cells. We don't have a conclusive answer why this occurs. We tested the hypothesis that WT animals may have preformed antibodies against ALPP because they may have been preexposed to ALPP by contact with $\operatorname{Tg}(\mathrm{ALPP})$ littermates. However, by using Coombs test and flow cytometry, we found no evidence of preformed anti-ALPP antibodies in naive WT animals. Therefore, it remains unclear why intraarticularly injected ALPP-expressing MSC are so rapidly cleared in WT recipients. In any case, however, this problem can be overcome by use of our $\operatorname{Tg}\left(\mathrm{ALPP}^{\mathrm{m}}\right)$ model. The pronounced reduction in the number of ALPPexpressing MSC attached to the defects between 1 and 7 days after MSC injection in $\operatorname{Tg}(\mathrm{ALPP})$ recipients is most likely due to fluid flow-induced shearing forces caused by joint movement, which may remove cells not firmly attached to the cartilage matrix. The latter finding underscores that more efficient site-specific transplantation techniques are needed to fully exploit the therapeutic potential of MSC for cartilage regeneration.

For the further development of regenerative therapies based on autologous adult stem cells (1) or autologous induced pluripotent stem cells (34-36), appropriate animal models are urgently needed. The marker tolerance technology we describe allows unbiased long-term tracking of coisogeneic, genetically labeled cells and tissues, closely mimicking the biology in a human patient treated with autologous cells. Taken together, this technology may represent an important advance in our ability to track labeled cells in immunocompetent hosts and in the development of predictive and cost-effective preclinical test systems for regenerative treatment approaches.

In our study, we used this potentially novel technology to answer one of the most burning questions currently in MSC-based cartilage regeneration: whether intraarticularly injected MSC contribute to cartilage regeneration in vivo via a progenitor- or nonprogenitor-mediated mechanism. Our findings in the focal cartilage defect model clearly indicate that attachment of MSC within full-thickness cartilage defects is necessary for cartilage regeneration. However, the MSC within the defect only orchestrate regeneration by host-derived cells; they do not give rise to new chondrocytes for long-term regeneration.

This conclusion is corroborated by a recent first-in-man clinical trial (37). de Windt and coworkers (37) found that allogeneic MSCs transplanted together with autologous chondrons into cartilage defects in the knee stimulated cartilage regeneration, as evidenced by MRI and second-look arthroscopies. However, biopsies taken from the regenerated cartilage during second-look arthroscopies failed to provide evidence of donor-derived DNA, showing that the transplanted allogeneic MSC did not permanently engraft in the host tissue. It could be argued that the results of the latter study may have been influenced by immune-mediated rejection of allogeneic cells. However, taken together, the study by de Windt et al. (37) in humans and our experimental data in an unbiased long-term cell-tracking model make a strong case for the notion that MSC stimulate long-term cartilage regeneration mainly via a nonprogenitor mechanism. 
Of note in this context, our data suggest that at least some MSC attached to the defects undergo early chondrogenesis by forming aggregates highly expressing the chondrogenic master transcription factor SOX9 $(38,39)$. It is known that SOX9 promotes chondrogenic differentiation and suppresses hypertrophic and osteogenic differentiation $(40,41)$. Therefore, we hypothesize that the transplanted MSC secrete bioactive factors that prevent chondrocyte hypertrophy in neighboring, host-derived cells. Hence, it is conceivable that the long-term overall role of MSC as orchestrators of endogenous cartilage regeneration may be coupled to a transient progenitor function. Future studies are needed to shed more light on the molecular nature of the crosstalk between transplanted MSC and host-derived cells.

\section{Methods}

Supplemental Methods are available online with this article.

Cloning of $A L P P$ and $A L P P^{m}$. The R26 promoter was amplified via PCR from genomic DNA extracted from F344-Tg(R26-ALPP) rats (16), and the genomic ALPP sequence including 3'UTR was amplified from human whole blood genomic DNA. PCR products R26S, E3r, R26f, U3Kr, U3Kf, and 3U1r (Supplemental Table 1) were cloned into pCR2.1 or pCR-4-TOPO (Invitrogen) and sequenced to verify mutation-free amplification. For E451G substitution, splice overlap extension (SOE) was performed using the primers shown in Supplemental Table 2. First, the 3'UTR fragment U3Kf/3Ur was cloned with KpnI and SpeI into a modified pEGFP-N3 vector (Clontech-Takara) where the CMV promoter and EGFP had been removed by AscI/NheI and KpnI/XbaI digestion, resulting in plasmid $\mathrm{p} \Delta \mathrm{N} 3-3$ 'UTR. This plasmid was opened by SalI/ KpnI, and the PCR fragments R26S/E3r and R26f/U3Kr were inserted via SalI, BamHI, and KpnI restriction sites to complete the R26P-ALPP construct. For the R26P-ALPPE451G construct, a PCR amplicon carrying the point mutation introduced by SOE was digested with Eco72I/KpnI and ligated into a pBSIIKS(-) vector opened by $\mathrm{KpnI} / \mathrm{BamHI}$ digestion, together with the BamHI/Eco72I-digested R26f/U3Kr-derived fragment. Finally, the R26P-ALPPE451G construct was completed by analogous assembly of fragments in $\mathrm{p} \Delta \mathrm{N} 3-3$ 'UTR via SalI, BamHI, and KpnI restriction sites. Final R26P-ALPP and R26P-ALPP ${ }^{\mathrm{m}}$ cassettes were sequenced prior to pronuclear injection.

Generation of transgenic mice and rats. The R26P-ALPP and R26P-ALPP ${ }^{\mathrm{E} 29 \mathrm{G}}$ cassettes were excised from the vector backbone using SalI and AfIII, separated by agarose gel electrophoresis, and recovered from gel using the QIAquick gel extraction kit (Qiagen). Pronuclear injections into fertilized oocytes derived from C57BL/6N or F344 (Charles River Laboratories) matings were performed as described (42). The purified DNA constructs were injected into a pronucleus at a concentration of $2.0 \mathrm{ng} / \mu 1$ injection buffer. Injected zygotes were transferred the same day into pseudopregnant surrogate mothers. To identify transgenic founder animals, the offspring were genotyped by Southern blot $(0.8 \mathrm{~kb}$ probe $)$ and PCR analysis using specific primers (Supplemental Table 2). Based on transgene expression pattern (see below) and the presence of only 1 insertion site, 2 donor (C57BL/6N-Tg[R26-ALPP]181Biat and C57BL/6N-Tg[R26-ALPP]188Biat) and 3 recipient mouse lines (C57BL/6N-Tg[R26-ALPP $\left.{ }^{\mathrm{E} 451 \mathrm{G}}\right]$ 192Biat, C57BL/6N-Tg[R26-ALPP $\left.{ }^{\mathrm{E} 451 \mathrm{G}}\right] 195 \mathrm{Biat}$, and C57BL/6N-Tg[R26-ALPPE451G]196Biat), and 1 donor (F344-Tg[R26-ALPP]Mhm) and 1 recipient rat line (F344-Tg[R26-ALPP $\left.{ }^{\mathrm{E} 451 \mathrm{G}}\right] 545 \mathrm{Mhm}$ ) were selected for further analysis.

$R N A$ isolation, $c D N A$ synthesis, and RT-PCR. For RNA isolation, tissue ( $n \geq 7$ per genotype) shock-frozen in liquid nitrogen and stored at $-80^{\circ} \mathrm{C}$ was homogenized in TRI Reagent (Ambion) in a MagNA Lyser Instrument (Roche Diagnostis). RNA was extracted with 1-bromo-3-chloropropane (MilliporeSigma) and precipitated using isopropanol. RNA purity and quality was determined spectrophotometrically (BioPhotometer, Eppendorf) and via agarose gels. Reverse transcription of RNA was performed using the iScript cDNA Synthesis Kit (Bio-Rad). Full-length expression of the transgenes was determined by several PCR assays using primers (Supplemental Table 2) spanning the entire transcript.

Immunoblotting. Frozen mouse lung ( $n \geq 7$ per genotype), rat kidney ( $n=4$ per genotype) and rat joint capsule samples ( $n \geq 5$ per group) were homogenized in $0.25 \mathrm{M}$ Tris- $\mathrm{HCl}, \mathrm{pH} 6.8$, using a MagNA Lyser Instrument (Roche Diagnostics) and diluted 1:1 with 6\% SDS. Protein samples were separated by SDSPAGE, and immunoblotting was carried out using a rabbit anti-ALPP antibody (SP15, Thermo Fisher Scientific), followed by incubation with HRP-conjugated goat anti-rabbit antibody (RPN4301, Amersham) and development with Clarity Western ECL Blotting Substrate (Bio-Rad). A rabbit anti-GAPDH antibody (MAB374, MilliporeSigma) was used as loading control and for normalization.

Skin transplantation. For skin transplantation, $0.5 \times 0.5 \mathrm{~cm}$ full mouse skin allografts and $1 \times 1 \mathrm{~cm}$ full rat skin allografts were transplanted from donors to recipients under isoflurane anesthesia $(n=10$ per 
group). In each animal, an autograft served as control. Oral metamizole administered before surgery and every 6 hours after surgery was used for analgesic therapy. Antibiosis was performed over 4 days after surgery using enrofloxacin (Baytril; $10 \mathrm{mg} / \mathrm{kg}$ s.c. once daily for mice, $20 \mathrm{mg} / \mathrm{kg}$ s.c. once daily for rats). Photographs of the grafts were taken every week after transplantation, and mice were killed 3 and 24 weeks after surgery by exsanguination from the vena cava under ketamine (Ricter Pharma)/xylazine (EuroVet Animal Health) anesthesia (70/7 mg/kg i.p.). Rats were killed 4 weeks after transplantation under $\mathrm{CO}_{2}$.

Histochemical and IHC analysis. Tissue samples from heart, lung, liver, kidney, spleen, vertebra, knee joints ( $n \geq 7)$, skin, and skin transplants from mice and rats ( $n=10$ per group) were harvested, fixed in $40 \%$ $\mathrm{EtOH}$, and processed for paraffin or a modified methylmethacrylate embedding (43). Sections (5- $\mu \mathrm{m}$ thick) were mounted on 3-aminopropyltriethoxy-silane (MilliporeSigma) pretreated slides, rehydrated, and heated at $72^{\circ} \mathrm{C}$ for 35 minutes to block both endogenous AP and ALPPm activity. Sections were then incubated in TNM buffer (0.1 M Tris- $\left.\mathrm{HCl}, \mathrm{pH} 9.5,0.1 \mathrm{M} \mathrm{NaCl}, 5 \mathrm{mM} \mathrm{MgCl}_{2}\right)$ containing $0.17 \mu \mathrm{g} / \mathrm{ml}$ of the substrate 5-bromo-4-chloro-3-indolyl phosphate (BCIP; MilliporeSigma) and $0.45 \mu \mathrm{g} / \mathrm{ml}$ nitrotetrazolium blue chloride (NBT; MilliporeSigma) at room temperature overnight to detect ALPP activity. Subsequently, sections were counterstained with methyl green (Vector), dehydrated, and mounted using Vectamount (Vector). Isolated whole distal femurs including the joint capsule were fixed with acetone- $\mathrm{MeOH}(30: 70 \mathrm{v} / \mathrm{v})$ for 10 minutes at $-20^{\circ} \mathrm{C}$, heated at $72^{\circ} \mathrm{C}$ for 75 minutes, stained with BCIP/NBT in TNM buffer for 3 hours at room temperature, and immediately analyzed under the microscope. ALPP-stained area in the defects and the synovium were quantified using ImageJ (NIH) as described in detail earlier (8). Toluidine blue staining was performed according to routine histological procedures. In brief, dissected femurs were embedded in frozen section compound (FSC 22; Leica) and snap-frozen in liquid nitrogen. Frozen samples were sectioned at $5 \mu \mathrm{m}$ thickness, using the tape method (26). Cryosections were fixed in ice-cold ethanol for 2 minutes, washed for 5 minutes, stained with Toluidine blue O (MilliporeSigma) for 15 minutes, dehydrated in ethanol for 30 seconds, and mounted in Vectamount (Vector). For evaluation of immune-mediated rejection, sections were rehydrated and stained for CD45R using a rat anti-CD45R (550286, BD Pharmingen) in mice or for CD45 using a mouse anti-CD45 antibody (550566, BD Pharmingen) in rats, biotinylated rabbit anti-rat IgG (BA-4001, Vector Labs), Vectastain Elite ABC Kit (Vector Labs), and 3,3'-Diaminobenzidine tetrahydrochloride hydrate (MilliporeSigma), dehydrated, and mounted using Eukitt. Positive cells were enumerated per tissue section ( $n \geq 3$ per animal).

Focal cartilage defects. Full-thickness cartilage defects with a $0.7-\mathrm{mm}$ diameter were placed in the patellofemoral groove of the right knee of male recipient animals at the age of $4-6$ months ( $n \geq 5$ per group) with an NIH Style Neuro Punch (Fine Science Tools [FST]) under isoflurane anesthesia. After placing the defect, the joint capsule and muscle were closed using 6-0 Vicryl (Ethicon), and the skin was closed using 4-0 Vicryl. The left knee served as internal intact control. Oral metamizole administered before surgery and every 6 hours after surgery was used for analgesic therapy. Antibiosis was performed over 5 days after surgery using enrofloxacin (Baytril; $20 \mathrm{mg} / \mathrm{kg}$ s.c. once daily). Seven days after surgery, the skin sutures were removed.

MSC culture and injection. MSC for injection were isolated from BM of 4- to 6-week-old male ALPP-transgenic $\mathrm{F} 344$ rats and cultured under hypoxic conditions $\left(37^{\circ} \mathrm{C}, 5 \% \mathrm{CO}_{2}\right.$, and $\left.3 \% \mathrm{O}_{2}\right)$ up to passage 5 as described in detail before (20). For intraarticular injection, $\operatorname{Tg}(\mathrm{ALPP})$ MSC were harvested, carefully washed with PBS (without calcium and magnesium, Lonza), and resuspended in native rat serum isolated from whole blood. 1 $\times 10^{7}$ cells were resuspended in $50 \mu 1$ rat serum per knee and injected intraarticularly with a $100-\mu 1$ syringe and a $23 \mathrm{G}$ needle under isofluorane anesthesia 2 weeks after surgery. The rat's knee was kept in a bent position, and the MSC were injected at a $90^{\circ}$ angle from the medial side in the space between femur, tibia, and patella. One day, 7 days, 1 month, and 6 months after injection, recipient rats were killed using $\mathrm{CO}_{2}$.

Immunofluorescence analysis. Immunofluorescence analysis was performed on cryosections of femurs using the tape method (26). Sections (5- $\mu \mathrm{m}$ thick) were fixed with ice-cold $\mathrm{MeOH}$ for 2 minutes at $-20^{\circ} \mathrm{C}$ and blocked with blocking buffer $(0.5 \%$ saponin, $2.5 \% \mathrm{MeOH}, 2 \%$ normal goat serum in $\mathrm{PBS}, \mathrm{pH} 7.4)$ for 45 minutes at room temperature. Cryosections were stained with the following primary antibodies: mouse anti-COL2 antibody (CP18, Calbiochem), rabbit anti-Sox9 antibody (AB5535, Millipore), chicken anti-COMP antibody (ab28400, Abcam), and mouse or rabbit anti-ALPP antibody (GTX72989, GeneTex; CBL207, Chemicon) in parallel. Thereafter, sections were incubated with a mixture of FITC-conjugated goat anti-mouse (F8771, MilliporeSigma), Alexa Fluor 555-conjugated goat anti-rabbit (4413, Cell Signaling Technology) or Cy3-conjugated goat anti-rabbit (111-165-144, Jackson ImmunoResearch), and Alexa 
Fluor 488-conjugated goat anti-chicken (ab150169, Abcam) for 1 hour at room temperature and mounted using Mowiol 4-88 (MilliporeSigma). DAPI (4083S, Cell Signaling) was used for nuclear staining. COL2 staining within the defect was quantified as percent of defect area by ImageJ ( $n=6$ sections per animal).

Statistics. All data are given as mean \pm SEM. Statistics were computed using IBM SPSS Statistics 20. Normal distribution of data was analyzed by Kolmogorov-Smirnov test. For 2 independent groups, 2-tailed Student's $t$ test was used. Data from more than 2 groups were analyzed using 1-way ANOVA, followed by Student-Newman-Keuls multiple comparison test, or by using Kruskal-Wallis test followed by Mann-Whitney $U$ test, as appropriate. $P<0.05$ were considered significant.

Study approval. All animal studies were approved by the Ethical Committee of the University of Veterinary Medicine, Vienna; by the Austrian Federal Ministry of Science and Research; and by the Regierungspräsidium Karlsruhe, Germany (permit nos. BMBWK-68.205/0240-BrGT/2005, BMWF-68.205/0018II/10b/2010, BMWF-68.205/0241-II/10b/2008, BMWF-68.205/0013-II/3b/2014, 35-9185.81/G-44/10) and were undertaken in strict accordance with prevailing guidelines for animal care and welfare.

\section{Author contributions}

DZ, VP, TR, and RGE designed research; DZ, MS, VP, JRG, KIO, SCH, TR, and MF performed research; DZ, MS, VP, JRG, KIO, and MF analyzed data; and DZ, MS, VP, TR, and RGE wrote the paper.

\section{Acknowledgments}

We thank C. Bergow, A. Rych, S. Kreidl, and C. Schüler for excellent technical assistance; M. Müller and A. Saalmüller for helpful discussions; and G. Tebb for critically reading the manuscript. This research was funded by grants from the University of Veterinary Medicine, Vienna, by the Austrian Science Fund FWF (P 21903-B13) to RGE and by the GEN-AU program Austromouse to TR.

Address correspondence to: Reinhold G. Erben, Institute of Physiology, Pathophysiology, and Biophysics, Department of Biomedical Sciences, University of Veterinary Medicine Vienna, Veterinärplatz 1, 1210 Vienna, Austria. Phone: 43.1.250.77.4550; Email: Reinhold.Erben@vetmeduni.ac.at.

DZ's present address is: Department for Medical Biochemistry, Max F. Perutz Laboratories (MFPL), Medical University Vienna, Vienna, Austria.

JRG's present address is: Department of Biomedical Sciences, Ross University School of Veterinary Medicine, St. Kitts and Nevis.

1. Zaher W, Harkness L, Jafari A, Kassem M. An update of human mesenchymal stem cell biology and their clinical uses. Arch Toxicol. 2014;88(5):1069-1082.

2. Agung M, et al. Mobilization of bone marrow-derived mesenchymal stem cells into the injured tissues after intraarticular injection and their contribution to tissue regeneration. Knee Surg Sports Traumatol Arthrosc. 2006;14(12):1307-1314.

3. Toupet $\mathrm{K}$, et al. Long-term detection of human adipose-derived mesenchymal stem cells after intraarticular injection in SCID mice. Arthritis Rheum. 2013;65(7):1786-1794.

4. Murphy JM, Fink DJ, Hunziker EB, Barry FP. Stem cell therapy in a caprine model of osteoarthritis. Arthritis Rheum. 2003;48(12):3464-3474.

5. Lee KB, Hui JH, Song IC, Ardany L, Lee EH. Injectable mesenchymal stem cell therapy for large cartilage defects--a porcine model. Stem Cells. 2007;25(11):2964-2971.

6. Wakitani S, Imoto K, Yamamoto T, Saito M, Murata N, Yoneda M. Human autologous culture expanded bone marrow mesenchymal cell transplantation for repair of cartilage defects in osteoarthritic knees. Osteoarthr Cartil. 2002;10(3):199-206

7. Bianco P, et al. The meaning, the sense and the significance: translating the science of mesenchymal stem cells into medicine. Nat Med. 2013;19(1):35-42.

8. Zwolanek D, Flicker M, Kirstätter E, Zaucke F, van Osch GJ, Erben RG. $\beta 1$ Integrins Mediate Attachment of Mesenchymal Stem Cells to Cartilage Lesions. Biores Open Access. 2015;4(1):39-53.

9. Heim DA, et al. Introduction of a xenogeneic gene via hematopoietic stem cells leads to specific tolerance in a rhesus monkey model. Mol Ther. 2000;1(6):533-544.

10. Rosenzweig M, et al. Induction of cytotoxic T lymphocyte and antibody responses to enhanced green fluorescent protein following transplantation of transduced CD34(+) hematopoietic cells. Blood. 2001;97(7):1951-1959.

11. Sarukhan A, Soudais C, Danos O, Jooss K. Factors influencing cross-presentation of non-self antigens expressed from recombinant adeno-associated virus vectors. J Gene Med. 2001;3(3):260-270.

12. Andersson G, et al. Nonmyeloablative conditioning is sufficient to allow engraftment of EGFP-expressing bone marrow and subsequent acceptance of EGFP-transgenic skin grafts in mice. Blood. 2003;101(11):4305-4312. 
13. Gross DA, Leboeuf M, Gjata B, Danos O, Davoust J. CD4+CD25+ regulatory T cells inhibit immune-mediated transgene rejection. Blood. 2003;102(13):4326-4328

14. Erben RG, et al. White paper on how to go forward with cell-based advanced therapies in Europe. Tissue Eng Part A. 2014;20(19 20):2549-2554.

15. Odörfer KI, Unger NJ, Weber K, Sandgren EP, Erben RG. Marker tolerant, immunocompetent animals as a new tool for regenerative medicine and long-term cell tracking. BMC Biotechnol. 2007;7:30

16. Kisseberth WC, Brettingen NT, Lohse JK, Sandgren EP. Ubiquitous expression of marker transgenes in mice and rats. Dev Biol. 1999;214(1):128-138.

17. Broekema M, et al. Determinants of tubular bone marrow-derived cell engraftment after renal ischemia/reperfusion in rats. Kidney Int. 2005;68(6):2572-2581.

18. Liu H, Guz Y, Kedees MH, Winkler J, Teitelman G. Precursor cells in mouse islets generate new beta-cells in vivo during aging and after islet injury. Endocrinology. 2010;151(2):520-528.

19. Unger NJ, Odörfer KI, Weber K, Sandgren EP, Erben RG. Utility of human placental alkaline phosphatase as a genetic marker for cell tracking in bone and cartilage. Histochem Cell Biol. 2007;127(6):669-674.

20. Balmayor ER, Flicker M, Käser T, Saalmüller A, Erben RG. Human placental alkaline phosphatase as a tracking marker for bone marrow mesenchymal stem cells. Biores Open Access. 2013;2(5):346-355.

21. Watanabe S, Watanabe T, Li WB, Soong BW, Chou JY. Expression of the germ cell alkaline phosphatase gene in human choriocarcinoma cells. J Biol Chem. 1989;264(21):12611-12619.

22. Watanabe T, Wada N, Kim EE, Wyckoff HW, Chou JY. Mutation of a single amino acid converts germ cell alkaline phosphatase to placental alkaline phosphatase. J Biol Chem. 1991;266(31):21174-21178.

23. Zambrowicz BP, Imamoto A, Fiering S, Herzenberg LA, Kerr WG, Soriano P. Disruption of overlapping transcripts in the ROSA beta geo 26 gene trap strain leads to widespread expression of beta-galactosidase in mouse embryos and hematopoietic cells. Proc Natl Acad Sci USA. 1997;94(8):3789-3794.

24. Hedrich HJ. Testing for Isohistogeneity (Skin Grafting). In: Adams M Hedrich HJ, eds. Genetic Monitoring of Inbred Strains of Rats: A Manual on Colony Management, Basic Monitoring Techniques, and Genetic Variants of the Laboratory Rat. New York: John Wiley \& Sons Inc; 1990:102-114.

25. Kolaczkowska E, Kubes P. Neutrophil recruitment and function in health and inflammation. Nat Rev Immunol. 2013;13(3):159-175.

26. Hong SH, Jiang X, Chen L, Josh P, Shin DG, Rowe D. Computer-Automated Static, Dynamic and Cellular Bone Histomorphometry. J Tissue Sci Eng. 2012;Suppl 1:004.

27. Andersson G, et al. Engraftment of retroviral EGFP-transduced bone marrow in mice prevents rejection of EGFP-transgenic skin grafts. Mol Ther. 2003;8(3):385-391.

28. Matsuo S, Kurisaki A, Sugino H, Hashimoto I, Nakanishi H. Analysis of skin graft survival using green fluorescent protein transgenic mice. J Med Invest. 2007;54(3-4):267-275.

29. Odörfer KI, Walter I, Kleiter M, Sandgren EP, Erben RG. Role of endogenous bone marrow cells in long-term repair mechanisms after myocardial infarction. J Cell Mol Med. 2008;12(6B):2867-2874.

30. Odörfer KI, et al. Hematopoietic bone marrow cells participate in endothelial, but not epithelial or mesenchymal cell renewal in adult rats. J Cell Mol Med. 2011;15(10):2232-2244.

31. Ivics Z, et al. Germline transgenesis in rabbits by pronuclear microinjection of Sleeping Beauty transposons. Nat Protoc. 2014;9(4):794-809.

32. Ivics Z, et al. Germline transgenesis in pigs by cytoplasmic microinjection of Sleeping Beauty transposons. Nat Protoc. 2014;9(4):810-827.

33. Chan AW, Chong KY, Martinovich C, Simerly C, Schatten G. Transgenic monkeys produced by retroviral gene transfer into mature oocytes. Science. 2001;291(5502):309-312.

34. Nishikawa S, Goldstein RA, Nierras CR. The promise of human induced pluripotent stem cells for research and therapy. Nat Rev Mol Cell Biol. 2008;9(9):725-729.

35. Yamanaka S. Strategies and new developments in the generation of patient-specific pluripotent stem cells. Cell Stem Cell. 2007;1(1):39-49.

36. Takahashi K, et al. Induction of pluripotent stem cells from adult human fibroblasts by defined factors. Cell. 2007;131(5):861-872

37. de Windt TS, Vonk LA, Slaper-Cortenbach ICM, Nizak R, van Rijen MHP, Saris DBF. Allogeneic MSCs and Recycled Autologous Chondrons Mixed in a One-Stage Cartilage Cell Transplantion: A First-in-Man Trial in 35 Patients. Stem Cells. 2017;35(8):1984-1993.

38. Lefebvre V, de Crombrugghe B. Toward understanding SOX9 function in chondrocyte differentiation. Matrix Biol. 1998;16(9):529-540

39. Hardingham TE, Oldershaw RA, Tew SR. Cartilage, SOX9 and Notch signals in chondrogenesis. J Anat. 2006;209(4):469-480.

40. Ikeda T, et al. The combination of SOX5, SOX6, and SOX9 (the SOX trio) provides signals sufficient for induction of permanent cartilage. Arthritis Rheum. 2004;50(11):3561-3573.

41. Akiyama H, et al. Interactions between Sox 9 and beta-catenin control chondrocyte differentiation. Genes Dev 2004;18(9):1072-1087.

42. Rülicke T. Pronuclear microinjection of mouse zygotes. Methods Mol Biol. 2004;254:165-194

43. Erben RG. Embedding of bone samples in methylmethacrylate: an improved method suitable for bone histomorphometry, histochemistry, and immunohistochemistry. J Histochem Cytochem. 1997;45(2):307-313. 